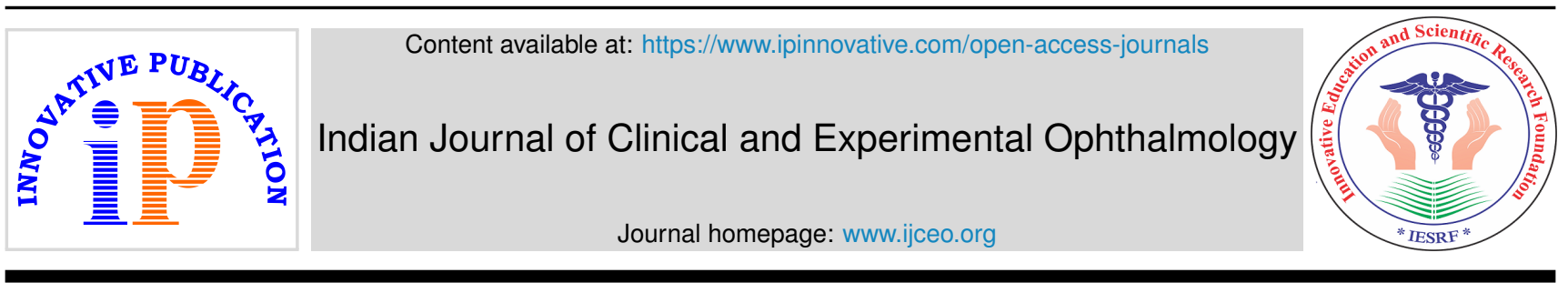

Original Research Article

\title{
Study of accuracy of axial length measurements by two biometric methods with reference to post operative spherical equivalents in patients undergoing phacoemulsification
}

\author{
Hanumant R Mandlik ${ }^{1}$, Sakshi R Patil ${ }^{1, *}$, Ajit K Joshi ${ }^{1}$, Abhishek Kumar Tripathi ${ }^{1}$, \\ N Sai Divya ${ }^{1}$ \\ ${ }^{1}$ Dept. of Ophthalmology, Bharati Vidyapeeth (Deemed to be university) Medical College and Hospital, Sangli, Maharashtra, \\ India
}

\section{A R T I C L E I N F O}

\section{Article history:}

Received 06-05-2020

Accepted 15-05-2020

Available online 22-12-2020

\section{Keywords:}

Cataract

Emmetropia

Intraocular lens

Visual acuity

\begin{abstract}
A B S T R A C T
Introduction: Cataract surgery demands refractive accuracy. To achieve this, accurate biometry is required. So two biometry methods should be compared for accuracy.

Aim: To compare accuracy of immersion USG and optical biometry with reference to AXL measurement and postphaco refraction.

Materials and Methods: Prospective study was done involving 60 eyes of 60 patients undergoing phaco with foldable IOL implantation under LA. Biometry of Group A was done by immersion USG and that of group B was done by optical biometry (30 patients in each group). Detailed preop examination was done. Calculations of IOL power in all patients were done by using SRK/T formula. At 1 month f/up, spherical equivalent was noted. Using regression formula, emmetropic IOL powers and their differences with implanted IOL powers were calculated. Statistical analysis was done using unpaired t test.

Results: Mean AXL in group B (23.2157 mm) was slightly longer than that in group A $(23.2093 \mathrm{~mm})$, $\{\mathrm{P}=0.9754\}$. Mean spherical equivalent at 1 month f/up in group B (0.4583) was slightly lesser than that in group $\mathrm{A}(0.4625),\{\mathrm{P}=0.973\}$. Mean of differences between emmetropic IOL and actual IOL implanted was lesser in group $\mathrm{B}(0.6877)$, than that in group $\mathrm{A}(0.6932),\{\mathrm{P}=0.9762\}$.

Conclusion: Difference in mean AXL and mean postop spherical equivalent between two methods of biometry were not statistically significant. Immersion USG biometry has accuracy comparable to optical biometry.

(C) This is an open access article distributed under the terms of the Creative Commons Attribution License (https://creativecommons.org/licenses/by/4.0/) which permits unrestricted use, distribution, and reproduction in any medium, provided the original author and source are credited.
\end{abstract}

\section{Introduction}

Recently phacoemulsification is the most preferred method of cataract surgery. ${ }^{1}$ Refractive accuracy and spectacle free vision is the demand of present era.

To achieve postoperative refractive accuracy, precise biometry and proper intraocular lens (IOL) power calculation is essential. ${ }^{2}$ Recently there are great improvements in the biometry equipments and mathematical formulae to calculate accurate IOL power. ${ }^{1}$

\footnotetext{
* Corresponding author.

E-mail address: opthosakshi@gmail.com (S. R. Patil).
}

Inaccurate axial length Measurement, wrong IOL calculation formula, faulty placement of IOL, errors in IOL constant and improper keratometric readings are the main obstacles in achieving postoperative refractive accuracy. The analysis of postoperative refractive surprises shows that in $43 \%$ cases there are faulty biometric and in $67 \%$ cases wrong keratometric readings. If axial length (AXL) is wrongly measured by $1 \mathrm{~mm}$, it leads to error of 3-3.5 D in IOL power calculation. Also wrong Keratometric reading by $1 \mathrm{D}$ leads to error of 0.9 to $1 \mathrm{D}$ in IOL power calculation. ${ }^{2}$

Axial length can be calculated by two methods viz Ascan ultrasound biometry and optical biometry. There are 
two methods of A scan ultrasound biometry viz contact and immersion method. Out of which immersion method is considered to be more accurate as compared to contact method because of absence of error induced due to corneal compression in the former. ${ }^{3}$ But main disadvantage of both these methods is that they give erroneous results in patients with very short eyes and cases having posterior staphyloma. $^{3}$

Optical biometry being non contact procedure is more accurate than A scan ultrasound biometry. It also has advantage of good reproducibility and is easy to perform. ${ }^{4}$ But it can not measure AXL in cases of dense posterior sub capsular cataracts, mature cataracts and also in patients having vitreous hemorrhage, maculopathy, or retinal detachment, where only A scan ultrasound biometry is possible. ${ }^{4,5}$

Optical biometer is considered to be more accurate than ultrasound biometry. ${ }^{4}$ But the cost of optical biometer is very high, so it is not affordable to many practioners and institutes especially in developing countries. Hence they have to depend on ultrasound biometry for axial length measurement as optical biometer may not be available. Recently use of premium IOLs i.e. multifocal and torric IOLs is increasing. For premium IOLs accurate biometry is a must, which is generally done by optical biometry. Off late demand for premium IOLs in patients with denser cataract is increasing. As optical biometry is not possible in such cases, we have to rely on ultrasound biometry. ${ }^{4,5}$ On this background, this study is undertaken to compare two biometry methods viz immersion ultrasound and optical biometry for their accuracy in cases undergoing phacoemulisifiaction with foldable IOL implantation.

\section{Materials and Methods}

\subsection{Aim}

To compare accuracy of immersion USG and optical biometry with reference to AXLs measurement and postphacoemulsification refraction.

\subsection{Objectives}

1. Primary objective: To compare axial lengths by immersion USG and optical biometry.

2. Secondary objectives: To calculate post operative spherical equivalent at 30 days follow up along with to calculation of predicted emmetropic IOL power using regression formula.

\subsection{Study question}

Is there any significant difference in axial length measurement by immersion ultrasound and optical biometry?

\subsection{Study design}

A prospective observational study.

\subsection{Method}

This study was done at a tertiary hospital between 1st January to 30th March 2019, involving 60 eyes of 60 subjects with cataract who underwent phacoemulsification with foldable IOL implantation under local anaesthesia.

The Study was approved by Institutional Ethical committee. (IEC approval letter number- IEC/335/18, dated: 26/12/2018).

\subsection{Inclusion criteria}

1. The patients having senile cataract with corneal astigmatism of less than $1.5 \mathrm{D}$.

2. The patients of age group between 45 to 85 years.

3. Axial lengths in between $22 \mathrm{~mm}$ and $24.5 \mathrm{~mm}$.

\subsection{Exclusion criteria}

1. The patients with nuclear cataract more than grade 3 and dense posterior subcapsular cataract.

2. Patients with glaucoma and other vitreoretinal diseases.

3. Corneal opacity, traumatic cataract, pseudoexfoliation cases.

After getting written and informed consent from all study patients, two groups of 30 patients each were made. They were divided in two groups randomly i.e. even numbered patients in group A and odd numbered patients in group B.

Biometry of Group A was done by immersion ultrasound and that of group B was done by optical biometry method. Then they were examined in detail and posted for phacoemulsification with PCIOL implanataion under LA.

Data collection: Preoperatively axial lengths were measured and IOL powers were calculated and noted in all patients of each group. Subjective refraction was noted of each patient at 30th day follow up.

Biometry of group A: In Group A patients Immersion A-scan ultrasound was done in supine position. After putting proparacaine $(0.5 \%)$ eyedrop to anaesthetize the eye, Prager scleral shell made of plastic was placed on patient's cornea in between two eyelids. Then Balanced Salt Solution (BSS) was injected in the shell. It acts as coupling medium in between patient's cornea and A-scan probe. The patient was asked to fix at light source in the probe and AXL readings were taken. With acceptable standard deviation, 5 readings were taken and average reading was noted. Then keratometric readings measured by auto-refractokeratometer were entered, and IOL power was calculated.

Biometry of group B : In Group B patients optical biometry was done in a sitting position. After proper 
positioning, patient is asked to look steady in the machine without blinking and measurements were taken in phakic mode. It calculates IOL power automatically.

Preoperatively all patients were examined in detail, which includes visual acuity, intraocular pressure (IOP) measurement, anterior segment and fundus examination etc. Visual acuity (unaided and best corrected) was recorded by using Snellen's chart. IOP was measured with applanation tonometer. Detailed anterior segment examination was done using slit lamp to look for corneal lesions, type and grade of cataract. Fundus was examined by slit lamp with 90D lens to rule out any vitreoretinal disease.

Biometry of all the patients were done by single person to avoid bias. IOL calculation for all the patients were done by using Sanders Retzlaff Kraff/Theoretical (SRK/T) formula and optimized A constant. It is universally accepted third generation formula for range of AXLs from $22 \mathrm{~mm}$ to 24.50 mm. ${ }^{6,7}$ The IOL powers were selected by keeping target refraction nearest to emmetropia.

After routine investigations of all the patients like blood sugar level, ELISA for HIV, Australia antigen test, urine microscopic examination etc., they were posted for phacoemulsification. Xylocaine sensitivity skin test was done for each patient.Then after dilation of the pupil with mydriatic (Tropicamide $0.8 \%+$ phenyleprine $5 \%$ eyedrop), local anaesthesia(LA) was given by peribulbar block. Solution containing 2\% Xylocaine with adrenaline (1:200000) was dissolved with injection hyaluronidase (1500 International Units) and about 4-5 ml was injected in peribulbar space. All the surgeries were performed by single surgeon with same technique. After painting of the eye with $5 \%$ povidone iodine solution, draping was done. Then universal eye speculum applied and superotemporal limbal incision and corneal tunnel made with using 2.8 mm keratome. With MVR blade 2 sideports were made. Continuous Curvilinear Capsulorhexis (CCC) of $5.5 \mathrm{~mm}$ size done after staining with tryphan blue dye. Then both hydrodissection and hydrodelination were done and nucleus was rotated. Then nucleus was emulsified by stop and chop technique. Epinuclues and cortex removed by automated irrigation and aspiration system. Then single piece foldable IOL made up of hydrophilic acrylic was injected and dialed in the capsular bag. Remaining viscoelastic material was removed by automated irrigation and aspiration. Then intracameral moxifloxacin $(0.5 \%)$ was injected. Sideports were sealed by hydration and then mixture of dexamethasone and gentamycin $(0.5 \mathrm{ml}$ each) was injected subconjuctivally. Chloramphenicol eye ointment(10mg per gm)was instilled in eye and patching was done.

After 4 hours patch was removed and slit lamp examination was done. Then eye drop containing $\operatorname{moxifloxacin}(0.5 \%)$ and dexamethasone $(0.1 \%)$ was prescribed 2 hourly for 1 week and tapered weekly. Patients were examined on 7th day and 30th day for anterior segment finding and visual acuity. Best corrected visual acuity and refractive error were noted. Mild complications like iritis and striate keratitis were found in few patients, which were treated accordingly. At 30th day follow-up, glasses were prescribed to each patient according to autorefractometry and final subjective refraction.

By using following regression formula, predicted IOL power to achieve emmteropia was calculated for each patient.

$\mathrm{Po}=\mathrm{Pi}+1.5^{*} \mathrm{Rx}$,

Where Po $=$ Predicted IOL power that would have produced emmetropia, $\mathrm{Pi}=$ actual power of the implanted IOL, $\mathrm{Rx}=$ post-operative spherical equivalent, ${ }^{8,9}$ which is calculated by addition of half of the cylinder power to spherical power. ${ }^{10}$

Same regression formula was also used by Menezo et al. and Olsen $\mathrm{T}$ in their study. ${ }^{8,9}$

Then the difference between actual implanted IOL power and predicted emmetropic IOL power was calculated for each patient.

\subsection{Statistical analysis}

The data was compiled in MS excel sheet and SPSS-22 software was used to analyse the data. The mean and standard deviation of axial length, postoperative spherical equivalent and difference between IOL powers placed and predicted Emmetropic IOL were calculated at 30th postoperative day in two groups.

Then all the variables were compared by using Unpaired t-test and $\mathrm{p}$ values were obtained.

\section{Results}

Present study included 60 eyes of 60 patients, which consists of 29 males and 31 females with ratio of $0.93: 1$. Group A consists of more females than group B.

Table 1: Gender wise distribution

\begin{tabular}{lccc}
\hline & Group A & Group B & Total \\
Male & $13(43.33 \%)$ & $16(53.33 \%)$ & $29(48.33 \%)$ \\
Female & $17(56.66 \%)$ & $14(46.66 \%)$ & $31(51.66 \%)$ \\
Total & 30 & 30 & $60(100 \%)$ \\
\hline
\end{tabular}

Maximum patients (24 i.e.40\%) were within the range of 56-65 years, out which group A had 13 and group B had 11 patients.(Table 2)

The distribution of patients according to preoperative and postoperative BCVA is shown in Table 3. Most (36 i.e. $60 \%$ ) of the patients had preoperative BCVA between $6 / 24$ to $6 / 60$. At 30th postoperative day most of the patients $(91.66 \%)$ had BCVA improved to $6 / 6$ to $6 / 9$.

The mean AXL in group B i.e. by optical biometry $(23.2157 \mathrm{~mm})$ was slightly longer than that in group A i.e.by 
Table 2: Age wise distribution

\begin{tabular}{lccc}
\hline Age in years & Group A & Group B & Total \\
$45-55$ & 06 & 08 & $14(23.33 \%)$ \\
$56-65$ & 13 & 11 & $24(40 \%)$ \\
$66-75$ & 09 & 10 & $19(31.66 \%)$ \\
$76-85$ & 02 & 01 & $03(05 \%)$ \\
Total & 30 & 30 & $60(100 \%)$ \\
\hline
\end{tabular}

Table 3: BCVA preoperative and 30th postoperative day

\begin{tabular}{lcc} 
BCVA & $\begin{array}{c}\text { Preoperative (no. } \\
\text { of patients) }\end{array}$ & $\begin{array}{c}\text { 30th } \\
\text { Postoperative day } \\
\text { (no. of patients) }\end{array}$ \\
Less than $6 / 60$ & 05 & 00 \\
$6 / 24-6 / 60$ & 36 & 00 \\
$6 / 12-6 / 18$ & 19 & 05 \\
$6 / 9-6 / 6$ & 00 & 55 \\
Total & 60 & 60 \\
\hline
\end{tabular}

immersion ultrasound $(23.2093 \mathrm{~mm}),\{\mathrm{P}=0.9754\}$, which shows no statistically significant difference.

Table 4: Mean axial length compared in two groups:

\begin{tabular}{lcc}
\hline $\begin{array}{l}\text { Axial length (no. } \\
\text { of patients) }\end{array}$ & $\begin{array}{c}\text { Group A } \\
\text { (Immersion A } \\
\text { scan) }\end{array}$ & $\begin{array}{c}\text { Group B (optical } \\
\text { biometry) }\end{array}$ \\
Mean & $23.2093 \mathrm{~mm}$ & $23.2157 \mathrm{~mm}$ \\
SD & 0.7667 & 0.8164 \\
P value $=0.9754$ & & \\
\hline
\end{tabular}

The Mean spherical equivalent at 30th postoperative day in group B (0.4583) was slightly lesser than that in group $\mathrm{A}(0.4625),\{\mathrm{P}=0.973\}$, which shows no statistically significant difference [Table 5].

Table 5: Postoperative spherical equivalent in two groups:

\begin{tabular}{lcc}
\hline $\begin{array}{l}\text { Actual post } \\
\text { operative } \\
\text { spherical } \\
\text { refraction }\end{array}$ & $\begin{array}{c}\text { Group A } \\
\text { (Immersion A } \\
\text { scan) }\end{array}$ & $\begin{array}{c}\text { Group B (optical } \\
\text { biometry) }\end{array}$ \\
Mean & $0.4625 \mathrm{D}$ & \\
$\mathrm{SD}$ & 0.5161 & $0.4583 \mathrm{D}$ \\
$\mathrm{P}$ value $=0.973$ & & 0.4284 \\
\hline
\end{tabular}

The mean of differences between emmetropic IOL and actual IOL implanted was lesser in group B (0.6877), than that in group A (0.6932), $\{\mathrm{P}=0.9762\}$, which shows no statistically significant difference.

\section{Discussion}

For postoperative refractive accuracy biometry must be precise. In present study, a comparison was done between immersion Ascan biometry and optical biometry in patients undergoing phacomemulsification, by subjecting two groups of patients to two different biometric methods.
Table 6: Comparison of difference between the actual IOL placed and predicted emmetropic IOL

\begin{tabular}{lcc}
\hline $\begin{array}{l}\text { Difference between } \\
\text { actual IOL and } \\
\text { emmetropic IOL }\end{array}$ & $\begin{array}{c}\text { Group A } \\
\text { (Immersion A } \\
\text { Mean) }\end{array}$ & $\begin{array}{c}\text { Group B } \\
\text { (optical } \\
\text { biometry) }\end{array}$ \\
SD & $0.6932 \mathrm{D}$ & $0.6877 \mathrm{D}$ \\
$\mathrm{P}$ value $=0.9762$ & 0.7739 & 0.6364 \\
\hline
\end{tabular}

Similar other studies have compared two methods by subjecting same patients to both methods.

As a primary outcome, it was found that mean axial length of immersion ultrasound is lesser than optical biometry, which was similar to results of prior studies.

Rajan MS et al. noted axial length of $23.47 \pm 1.1 \mathrm{~mm}$ by optical biometry and $23.43 \pm 1.2 \mathrm{~mm}$ by ultrasound biometry. ${ }^{11}$ Nakhli FR et al. noted mean AXL was 23.76 $\pm 1.87 \mathrm{~mm}$ with optical biometry and $23.86 \pm 1.85 \mathrm{~mm}$ with applanation ultrasound. ${ }^{12}$

Wang XG et al. compared axial lengths by 3 methods in normal and high myopes. Two optical biometric methods namely IOL master and lenstar and aplannation ultrasound method were used to measure AXLs. They noted axial lengths $23.18 \pm 0.77 \mathrm{~mm}, 22.94 \pm 0.75 \mathrm{~mm}$ and $22.94 \pm 0.75$ $\mathrm{mm}$ respectively in normal eyes. ${ }^{13}$ Results are similar in our study i.e AXLs are $23.41 \pm 0.81 \mathrm{~mm}$ and $23.20 \pm 0.76 \mathrm{~mm}$ by optical and immersion USG respectively. So, all studies shows axial length by optical biometry is slightly higher than by ultrasound biometry, but the difference is not statically significant.

As a secondary outcome it was found postoperative mean spherical equivalent of immersion ultrasound group is higher than that of optical biometry.

Rajan MS et al. found the mean of postoperative spherical equivalents was $0.6 \pm 0.4$ dioptres in patients who underwent ultrasound biometry. The spherical equivalent in the optical biometry group was $0.52 \pm 0.35 \mathrm{D}$. They found that difference of postoperative refractive error in two groups was not statistically significant. In our study postoperative spherical equivalent was $0.46 \pm 0.51$ diopetrs in ultrasound biometry group and $0.45 \pm 0.42$ diopetrs in optical biometry group. Here also difference between the means of postoperative spherical equivalent in two groups was not statistically significant.

Gaballa SH et al. in their study, compared two biometry methods by calculating predicted IOL power and postoperative refractive error. ${ }^{13}$ They found that Mean Numerical Error measured by IOL master was $0.16 \pm 0.18$ $\mathrm{D}$ which was lesser than that by A-scan as $(0.17 \pm 0.43 \mathrm{D})$. Similarly this study also found that mean postoperative spherical equivalent in optical biometry $(0.45 \pm 0.42 \mathrm{D})$ is lesser than that in immersion $(0.46 \pm 0.51 \mathrm{D}) .{ }^{14}$

We also calculated targeted emmetropic IOL power by regression formula. Similar calculations were done by Joshi 
AK et al. in their study for 60 eyes of 60 patients who were subjected to SICS with foldable IOL implantation. ${ }^{15}$ The power of the IOL that would have resulted into no residual postoperative refraction is calculated using the formula $\mathrm{Po}=\mathrm{Pi}+1.5 \mathrm{Rx}$ which is an accurate and different way of calculating the predicted IOL power.

Mean difference between implanted IOL and emmetropic IOL was $0.69 \pm 0.77$ diopters in immersion ultrasound group and $0.68 \pm 0.63$ in optical biometry group, which was not statistically significant.

\subsection{Interpretation and implications}

The axial Length measurement by optical biometry is slightly longer than immersion ultrasound and difference in post operative refractions are not statistically significant when biometry is done on emmetropic axial lengths by optical or Ultrasound method. So, both methods can be used in clinical practice with equal accuracy.

\section{Limitations of the Study}

Sample size was relatively small and variables other than emmetropic axial length were not considered. Hence future research with larger sample size and with more variables like non emmetropic axial lengths, corneal astigmatism cases will further enhance our knowledge of strength and limitations of existing two methods of biometry.

\section{Conclusion}

In conclusion, immersion ultrasound biometry has accuracy comparable to optical biometry when used in the eyes AXLs ranging $22 \mathrm{~mm}$ to $24.50 \mathrm{~mm}$. Immersion ultrasound biometry is reliable and affording method for measuring AXL and it has special role in patients with dense ocular media where optical biometry is not possible. However the optical biometry is quick and easy to use and provides a noncontact technique with no risk of infection or corneal abrasion and most accepted by patients.

\section{Source of Funding}

None.

\section{Conflict of Interest}

The authors declare that there is no conflict of interest regarding the publication of this article.

\section{References}

1. Moeini HA, Eslami F, Rismanchian A, Akhlaghi MR, Najafian JA. Comparison of ultrasound and optic biometry with respect to eye refractive errors after phacoemulsification. J Res Med Sci. 2008;13(2):43-7.

2. Karabela Y, Eliacik M, Kocabora MS, Erdur SK, Baybora H Predicting the refractive outcome and accuracy of IOL power calculation after phacoemulsification using the SRK/T formula with ultrasound biometry in medium axial lengths. Clin Ophthalmol. 2017;11:1143-9. do1:10.2147/opth.s136884

3. Astbury N, Ramamurthy B. How to avoid mistakes in biometry. Community Eye Health. 2006;19(60):70-1.

4. Sahin A, Hamrah P. Clinically relevant biometry. Curr Opin Ophthalmolo. 2012;23(1):47-53.

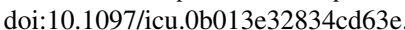

5. Mylonas G, Sacu S, Buehl W, Ritter M, Georgopoulos M, SchmidtErfurth U. Performance of three biometry devices in patients with different grades of age-related cataract. Acta Ophthalmol. 2011;89(3):e237-41. doi:10.1111/.1755-3768.2010.02042.x.

6. Biometry for Intra-Ocular Lens (IOL) power calculation-EyeWiki. [cited 10 April 2019]. Available from: https://eyewiki.aao.org/ Biometry_for_Intra-Ocular_Lens_(IOL)_power_calculation.

7. Overview | Cataracts in adults: management | Guidance | NICE [Internet]. Nice.org.uk. 2019 [cited 10 April 2019]. Available from: https://www.nice.org.uk/guidance/ng77.

8. Menezo JL, Chaques V, Harto M. The SRK regression formula in calculating the dioptric power of intraocular lenses. Br J Ophthalmol. 1984;68(4):235-7. do1:10.1136/bjo.68.4.235.

9. Olsen T. Calculation of intraocular lens power: A review. Acta Ophthalmol Scand. 2007;85(5):472-85.

10. Kolker RJ. Subjective Refraction and Prescribing Glasses: The Number One (or Number Two) Guide to Practical Techniques and Principles. JCAHPO; 2015.

11. Rajan MS, Keilhorn I, Bell JA. Partial coherence laser interferometry vs conventional ultrasound biometry in intraocular lens power calculations. Eye. 2002;16(5):552-6. do1:10.1038/s].eye.6700157.

12. Nakhli FR. Comparison of optical biometry and applanation ultrasound measurements of the axial length of the eye. Saudi J Ophthalmol. 2014;28(4):287-91. do1:10.1016/j.Sjopt.2014.04.003.

13. Wang XG, Dong J, Pu YL, Liu HJ, Wu Q. Comparison axial length measurements from three biometric instruments in high myopia. Int $\mathrm{J}$ Ophthalmol. 2016;9(6):876-80.

14. Gaballa SH, Allam RS, Abouhussein NB, Raafat KA. IOL master and A-scan biometry in axial length and intraocular lens power measurements. Delta J Ophthalmol. 2017;18(1):13-9. 601:0.4103/DO-9 73.201623.

15. Joshi AK, Tripathi AK, Parashar AD, Patil SR. Comparative Evaluation of Accuracy of Immersion A-scan Ultrasound Biometry and Optical Biometry in Cases Undergoing Small Incision Cataract Surgery. J Clin Diagn Res. 2019;13(7):4-8.

\section{Author biography}

Hanumant R Mandlik, Assistant Professor

Sakshi R Patil, Associate Professor

Ajit K Joshi, Professor and HOD

Abhishek Kumar Tripathi, Junior Resident

N Sai Divya, Junior Resident

Cite this article: Mandlik HR, Patil SR, Joshi AK, Tripathi AK, Divya NS. Study of accuracy of axial length measurements by two biometric methods with reference to post operative spherical equivalents in patients undergoing phacoemulsification. Indian J Clin Exp Ophthalmol 2020;6(4):595-599. 